Utah State University

DigitalCommons@USU

$1-1-2010$

\title{
The Magnetohydrodynamic Response of Liquid Oxygen: Experimentation and Simulation
}

\author{
J. C. Boulware
}

S. Wassom

S. Jensen

H. Ban

Follow this and additional works at: https://digitalcommons.usu.edu/sdl_pubs

\section{Recommended Citation}

Boulware, J. C.; Wassom, S.; Jensen, S.; and Ban, H., "The Magnetohydrodynamic Response of Liquid Oxygen: Experimentation and Simulation" (2010). Space Dynamics Lab Publications. Paper 22.

https://digitalcommons.usu.edu/sdl_pubs/22

This Article is brought to you for free and open access by the Space Dynamics Lab at DigitalCommons@USU. It has been accepted for inclusion in Space Dynamics Lab Publications by an authorized administrator of DigitalCommons@USU. For more information, please contact digitalcommons@usu.edu.

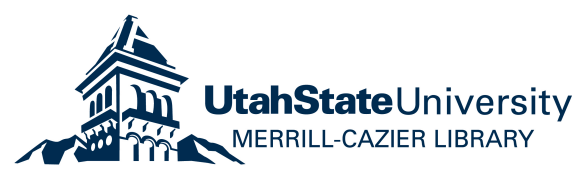




\section{AIP $\mid$ Proceedings}

\section{THE MAGNETOHYDRODYNAMIC RESPONSE OF LIQUID OXYGEN: EXPERIMENTATION AND SIMULATION}

J. C. Boulware, S. Wassom, S. Jensen, and H. Ban

Citation: AIP Conference Proceedings 1218, 1554 (2010); doi: 10.1063/1.3422336

View online: http://dx.doi.org/10.1063/1.3422336

View Table of Contents:

http://scitation.aip.org/content/aip/proceeding/aipcp/1218?ver=pdfcov

Published by the AIP Publishing

\section{Articles you may be interested in}

Numerical study of the formation process of ferrofluid droplets

Phys. Fluids 23, 072008 (2011); 10.1063/1.3614569

On the magnetohydrodynamic load and the magnetohydrodynamic metage

Phys. Plasmas 15, 012112 (2008); 10.1063/1.2836617

Effective magnetoviscosity of planar-Couette magnetic fluid flow

J. Appl. Phys. 97, 10Q302 (2005); 10.1063/1.1850337

A numerical study of the alpha model for two-dimensional magnetohydrodynamic turbulent flows

Phys. Fluids 17, 035112 (2005); 10.1063/1.1863260

Fluid pumped by magnetic stress

Appl. Phys. Lett. 86, 024102 (2005); 10.1063/1.1846956 


\title{
THE MAGNETOHYDRODYNAMIC RESPONSE OF LIQUID OXYGEN: EXPERIMENTATION AND SIMULATION
}

\author{
J. C. Boulware ${ }^{1}$, S. Wassom ${ }^{2}$, S. Jensen ${ }^{2}$, and H. Ban ${ }^{1}$ \\ ${ }^{1}$ Utah State University, Mechanical and Aerospace Engineering \\ Logan, UT, 84322, USA \\ ${ }^{2}$ Space Dynamics Laboratory \\ North Logan, UT, 84341, USA
}

\begin{abstract}
Experimental and theoretical studies have been conducted to establish the basic understanding and predictive capability for the dynamics of a liquid oxygen (LOX) slug subjected to magnetic fields within a solenoid. The electrically-pulsed solenoids around a $1.9 \mathrm{~mm}$ ID quartz tube were capable of producing up to $1.1 \mathrm{~T}$ when immersed in liquid nitrogen. The slug dynamics were measured by pressure changes in a closed volume on both sides of the slug. A theoretical model was developed which balances the magnetic, viscous, and pressure forces into a single equation of motion. The model was applied to a one-dimensional discretized algorithm that solved the coupled multiphysics problem of the Navier-Stokes and Maxwell's equations. The simulation and experimental results established LOX as a good candidate in a magnetic fluid system without moving parts for cryogenic applications.
\end{abstract}

KEYWORDS: Liquid oxygen, magnetohydrodynamics, magnetic fluids, ferrofluids.

\section{INTRODUCTION}

The strong paramagnetic susceptibility of liquid oxygen (LOX) has established it as a good candidate for a cryogenic magnetic fluid system void of mechanically moving parts. As a paramagnetic material, oxygen is attracted to an increasing magnetic field gradient by its susceptibility. Curie's Law states that paramagnetic susceptibility increases as temperature decreases and results from the reduced thermal energy allowing polar molecules to align with an external field. At cryogenic temperatures, oxygen liquefies whereas most other ferrofluids freeze; thus, LOX, which is already used extensively in 
propellant, thermal, and life support systems, becomes a good candidate for a cryogenic magnetic fluid system. Moving parts often limit a subsystem's lifetime due to their eventual failure and are particularly hindering in applications such as satellites where human intervention is not feasible. Therefore, a strong technical need exists for a quantitative database and predictive capability of the viability of LOX as a magnetic working fluid.

Past research on paramagnetic fluids such as LOX is limited. On the other hand, ferrofluids, which are colloidal suspensions of magnetic particles in a carrier fluid, have been heavily studied [1]. A ferrofluid pump capable of producing $2 \mathrm{kPa}(0.29 \mathrm{psi})$ was developed by Park and Seo [2-6] and others created by Krauss [7], Hatch [8], and Liao [9] were used as models of experimentation but did not generate as much pressure as Seo and Park's. As a paramagnetic fluid, LOX is about 30 times weaker than an inexpensive ferrofluid. Youngquist [10] performed the only quantitative experiments found on the magnetohydrodynamics (MHD) of LOX. With an open-ended U-tube, he measured the displacement of one end of a column of LOX while the other was pulsed by a solenoid. Youngquist also created a simulation that agreed well and, therefore, was used as a starting point for this study. In all, the current knowledge and understanding of MHD behavior of LOX are insufficient for the development of cryogenic applications. The objectives of this study were to displace a slug of LOX with electrically-pulsed solenoids and then develop a theoretical model to simulate LOX dynamics.

\section{EXPERIMENTAL SETUP}

An apparatus was designed to displace a slug of LOX via electrically-pulsed solenoids. The test section was completely submerged in liquid nitrogen to mitigate thermal acoustic oscillations. Helium acted as the surrounding gas for the LOX slug due to its inertness and low boiling point. A photograph and 3-dimensional drawing of the plumbing system can be seen in Figure 1. The total system volume was $340 \mathrm{~cm}^{3}$, although only about $1.8 \mathrm{~cm}^{3}$ existed downstream of the test section. This allowed for high-pressure changes when the slug was displaced. Pressure sensors upstream and downstream of the LOX slug in the test section recorded data at $5 \mathrm{kHz}$ with a $70 \mathrm{~Pa}$ resolution. The solenoids and test section were created by wrapping copper wire on plastic bobbins and then sliding them over a $1.9 \mathrm{~mm}$ ID quartz tube. The quartz tube interfaced with stainless steel plumbing

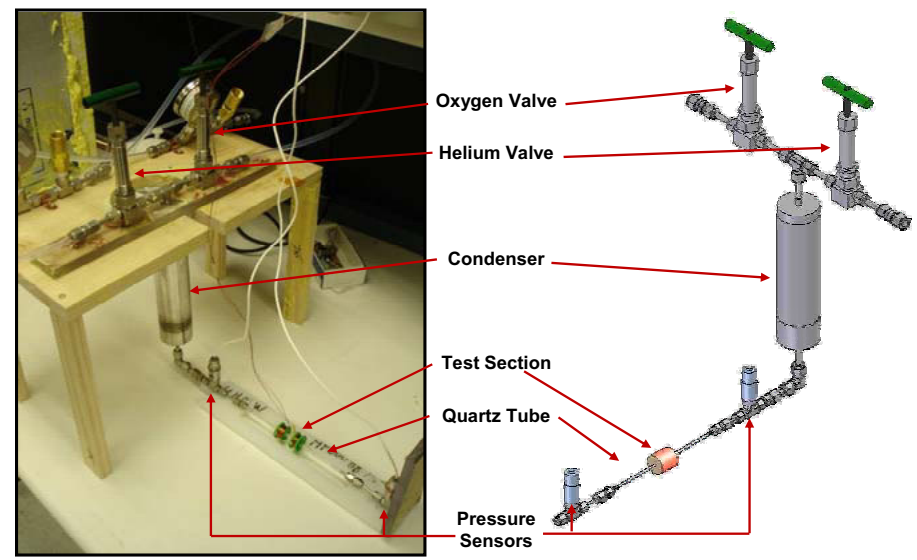

FIGURE 1. Photograph and CAD Drawing of Experimental Apparatus 
through removable Teflon heat shrink tubing that allowed for easy interchangeability of the test section. The maximum absolute pressure in the system did not exceed $172 \mathrm{kPa}$ and all fittings provided a helium-tight seal with leak rates less than $20 \mathrm{~Pa} / \mathrm{min}$. When submerged in liquid nitrogen, the solenoids produced up to $1.1 \mathrm{~T}$ with a $900 \mathrm{~W}$ power supply. The operating pressure was maintained above atmospheric pressure to prevent a cold vacuum, but held within a gauge pressure of $325 \mathrm{kPa}$ to maintain safe operating levels. Before submersion, the system was purged with helium and the direction of helium flow from upstream to downstream was the source of the notation used for convention. Prior to each test, the length of the slug and its initial position relative the solenoid were recorded. The LOX slug edges were measureable within $1.6 \mathrm{~mm}$ resolution.

\section{THEORY}

The dynamics of a LOX slug being displaced by magnetic fields was developed based on Rosensweig's analysis [1]. In a closed volume, the motion of the slug was similar to a pneumatic spring-mass-damper system whose forcing function could be described as a separable function of position and velocity. The position-based force on the slug was due to pressure $\left(F_{P}\right)$ and magnetic forces, $\left(F_{M}\right)$, and the velocity-based force was due to the wall shear force $\left(F_{S}\right)$

$$
m \ddot{x}=F_{P}(x)+F_{M}(x)+F_{S}(\dot{x})
$$

where $m$ is the slug mass; and $x, \dot{x}$ and $\ddot{x}$ is the position, velocity, and acceleration of the slug. The pressure force resulted from the differential pressure on either side of the slug as

$$
F_{P}=\pi a^{2} \Delta p
$$

where $a$ is the slug radius; and $\Delta p$ is the pressure difference. The magnetic force is based on Kelvin force density, $f_{m}$, as a result of the divergence of the Maxwell stress tensor and is

$$
f_{m}=\mu_{0}(\boldsymbol{M} \cdot \nabla) \boldsymbol{H}
$$

where $\mu_{0}$ is the permeability of free space, $\boldsymbol{M}$ is the magnetization vector, and $\boldsymbol{H}$ is the applied field vector. For soft magnetic materials, volumetric susceptibility is the ratio of the magnetization to the applied field, $\chi=\boldsymbol{M} / \boldsymbol{H}$. By substituting for $\boldsymbol{M}$, using the vector identity, $\boldsymbol{H} \cdot \nabla \boldsymbol{H}=\frac{1}{2} \nabla(\boldsymbol{H} \cdot \boldsymbol{H})-\boldsymbol{H} \times(\nabla \times \boldsymbol{H})$, and noting that Ampere's Law cancels out the curl of the applied field, equation (3) can be reduced to

$$
f_{m}=\frac{1}{2} \mu_{0} \chi \nabla \boldsymbol{H}^{2} .
$$

With a constant temperature, the relative permeability, $\mu$, also remains constant. The relative permeability is the ratio between the magnetic flux density, $\boldsymbol{B}$, and applied magnetic fields, $\mu=\boldsymbol{B} / \boldsymbol{H}$, but can also expressed in terms of volumetric susceptibility, $\mu=\mu_{0}(1+\chi)$. Given these relations, the Kelvin force density in the axial direction is

$$
f_{m, x}=\frac{1}{2 \mu_{0}} \frac{\chi}{(1+\chi)^{2}} \frac{d}{d x} B_{x}{ }^{2}
$$


where the subscript $x$ denotes the axial direction. The differential term considers the ends of the slug and when integrated over the entire volume of the slug, the force due to magnetism in the axial direction, $F_{M}$, is

$$
F_{M}=\frac{\pi a^{2}}{2 \mu_{0}} \frac{\chi}{(1+\chi)^{2}}\left(B_{x, U S}^{2}-B_{x, D S}^{2}\right)
$$

where the subscript $D S$ denotes the downstream edge of the slug; and $U S$ denotes the upstream edge. The magnetic flux density on the axis of a solenoid can be predicted by summing all the wire loops in the solenoid. The individual loop contribution is

$$
B_{x, \text { ind }}=\frac{1}{2} I \mu_{0} r^{2}\left(r^{2}+x^{2}\right)^{-3 / 2}
$$

where $I$ is the electrical current; $r$ is the wire radius; and $x$ is the axial distance to the center of the loop. Damping results from the force due to fluid shear on the wall, $F_{S}$, which is dependent on the wall shear stress of the transient LOX flow. As an approximation, steady state pipe flow shear stress was used with the classic relation for laminar wall shear stress, the Blasius correlation for turbulent wall shear stress [11], and an interpolative relation for the transient region as

$$
\begin{array}{ll}
F_{S}=2 \pi a L \tau_{W} & \\
\tau_{w}=4 \dot{x} \eta / a & \operatorname{Re}<2000 \\
\tau_{w}=\frac{\rho \dot{x}^{2}}{2}(0.0153 \log (\mathrm{Re})-0.0424) & 2000 \leq \mathrm{Re} \leq 3000 \\
\tau_{w}=0.0396 \rho^{3 / 4} \dot{x}^{7 / 4} \eta^{1 / 4}(2 a)^{-1 / 4} & \mathrm{Re}>3000
\end{array}
$$

where $\tau_{w}$ is the wall shear stress; Re is the Reynolds number; $\rho$ is the density of LOX; $\eta$ is the dynamic viscosity of LOX; and L is the length of the slug. When equations (2), (6), and (8) are substituted into equation (1), the equation of motion for the LOX slug becomes

$$
m \ddot{x}=\pi a^{2} \Delta p+\frac{\pi a^{2}}{2 \mu_{0}} \frac{\chi}{(1+\chi)^{2}}\left(B_{x, U S}^{2}-B_{x, D S}^{2}\right)+2 \pi a L \tau_{w} .
$$

\section{NUMERICAL METHOD}

The governing equations were discretized using a first-order finite-difference method for space and time. Given initial conditions of the position, velocity, and total force, a kinematic equation was used to find the slug's position at each time step. The discretized algorithms for calculating the pressure and magnetic forces are summarized in equations (11-15).

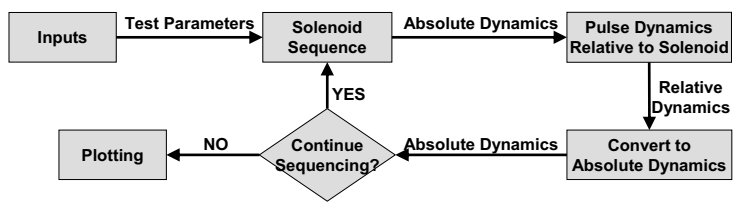

FIGURE 2. Numerical Simulation Block Diagram 


$$
\begin{gathered}
F_{P, i}=\pi a^{2}\left(P_{D S, i}-P_{U S, i}\right) \\
F_{S, i}=2 \pi a L \tau_{w, i} \\
B_{x, U S, i}=\frac{\mu_{0} I}{2} \sum_{m=-\frac{1}{2}\left(M_{x}-1\right)}^{\frac{1}{2}\left(M_{x}-1\right)} \sum_{n=0}^{M_{r}-1} \frac{\left(a_{s o l}+n b \Delta r\right)^{2}}{\left[\left(a_{s o l}+n b \Delta r\right)^{2}+\left(x_{i}-m b \Delta x\right)^{2}\right]^{3 / 2}} \\
B_{x, D S, i}=\frac{\mu_{0} I}{2} \sum_{m=-\frac{1}{2}\left(M_{x}-1\right)}^{\frac{1}{2}\left(M_{x}-1\right)} \sum_{n=0}^{M_{r}-1} \frac{\left(a_{s o l}+n b \Delta r\right)^{2}}{\left[\left(a_{s o l}+n b \Delta r\right)^{2}+\left(x_{i}-L-m b \Delta x\right)^{2}\right]^{3 / 2}} \\
F_{M, i}=\frac{\pi a^{2}}{2 \mu_{0}} \frac{\chi}{(1+\chi)^{2}}\left(B_{x, U S, i}{ }^{2}-B_{x, D S, i}{ }^{2}\right)
\end{gathered}
$$

where the subscript $i$ is the time step; $F_{T}$ is the total force on the slug from the pressure, magnetic, and shear components; $M_{x}$ and $M_{r}$ are the number of coils in the axial and radial directions; $\Delta z$ and $\Delta r$ are the coil spacing in the axial and radial directions; $a_{\text {sol }}$ is the inner radius of the solenoid, and $b$ is the wire diameter. The convention used was that positive displacement is in the upstream direction. With all three external forces determined, a total force on the slug could be formed.

$$
F_{T, i}=F_{P, i}+F_{M, i}-\operatorname{sgn}\left(\dot{x}_{i-1}\right) F_{S, i}
$$

where the sign function in the third term on the right hand side forced the shear component to always act in the opposite direction of velocity. Then, the left hand side of equation (10) was discretized and balanced with the average total force between time steps. After rearranging, the equation of motion was

$$
\dot{x}_{i}=\frac{F_{T, i}+F_{T, i-1}}{2 \rho \pi a^{2} L} \Delta t+\dot{x}_{i-1} .
$$

The numerical simulation then cycled to the next time step and repeated the algorithm to calculate the pulse dynamics relative to a solenoid. Once the total relative dynamics were solved for a solenoid, they were converted to absolute coordinates for the entire test setup. The process was repeated throughout the entire pulsing sequence as shown in Figure 2.

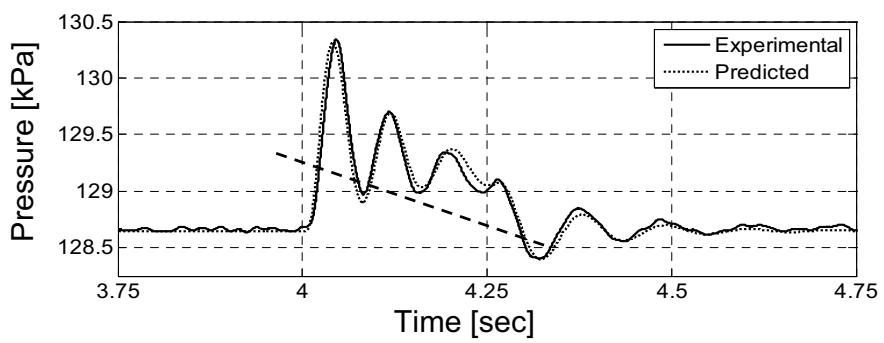

FIGURE 3. Downstream Pressure Response of a LOX Slug Propagated by an Electrically-Pulsed Solenoid. 


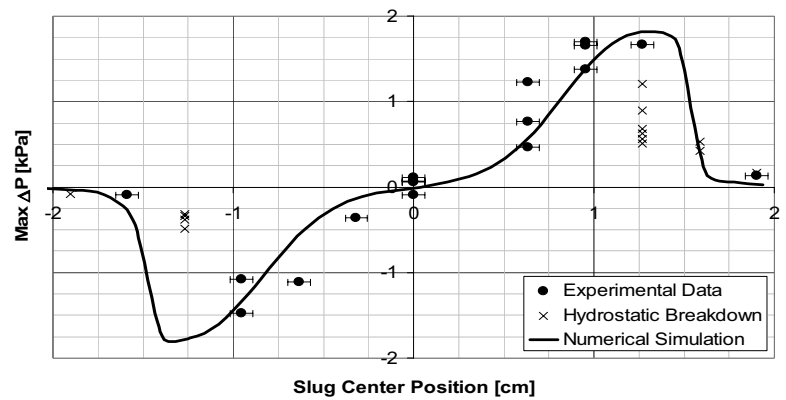

FIGURE 4. Experimental versus Numerical Pressure Generated for a $1.9 \mathrm{~cm}$ LOX Slug.

\section{RESULTS AND DISCUSSION}

The experiment produced interesting results for the slug dynamics. When power to the solenoids was switched on, the slug moved to a new position and oscillated about the mean displacement with decaying oscillation amplitude. The mean displacement decreased with time, corresponding to resistance heating that caused the magnetic field to decrease. The susceptibility decreased no more than $1 \%$ due to resistance heating. When power to the solenoid was switched off, the slug moved back to its neutral position and pressures on both sides of the slug equalized, again with damped oscillations. Positive pressure indicates the slug was displaced towards the downstream end causing compression and the dashed line represents the decreasing mean displacement when the solenoid is powered. Figure 3 shows the downstream pressure response and numerical simulation of a 0.25 second, $1.1 \mathrm{~T}$ pulse on a $1.9 \mathrm{~cm}$ slug, using an effective slug length of $5.3 \mathrm{~cm}$. The effective slug length is calculated by fitting the experimental data to the theoretical equations and a pneumatics analysis [12], but may actually be due to an improper experimental approximation. Because the metal tubing in upstream and downstream sections of the slug prohibit visual confirmation of the amount of LOX, additional slugs may have formed. If so, the dynamics of the system must include these slugs and an effective slug length larger than the visible slug length must be used for the numerical model. Further inabilities of the model include the lack of approximations for the magnetoviscous effects and slug internal flow.

To best match the experimental data, the run in Figure 4 assumed a viscosity 4.75 times that of non-magnetized LOX at $77 \mathrm{~K}$. The viscosity of LOX was augmented to
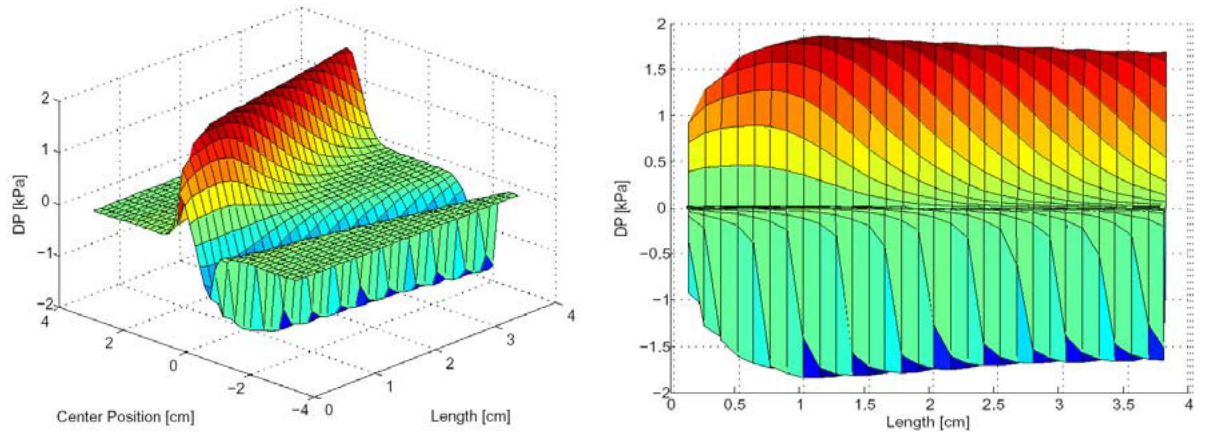

FIGURE 5. Pressure Dependency on Slug Length and Position 


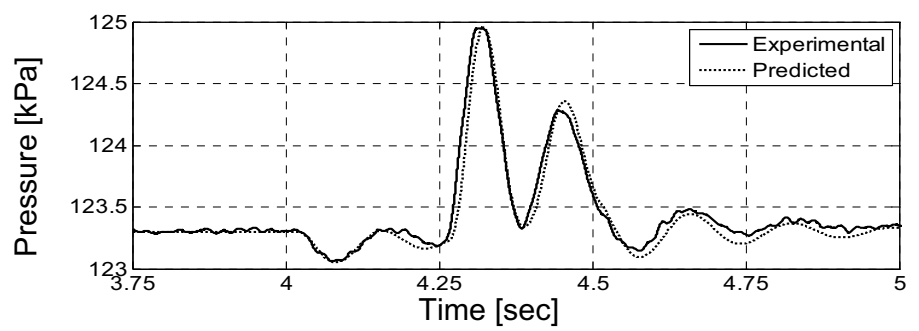

FIGURE 6. Downstream Pressure Correlation of Experimental Data and Numerical Simulation

account for unknown magnetoviscous effects and internal flow dynamics. It has long been known that a magnetic field will effect viscosity and Odenbach [13] has shown that accurately predicting the changes requires the use of laboratory measurements. Unfortunately, no data could be found for LOX. Unknown velocity gradients of the internal flow at the wall could have also resulted in greater shear stress. For the simplicity of the current model, the viscosity was calculated based on the best fit of the data to the slug motion equations. Further studies of the phenomena using cryogenic rheometers and computational fluid dynamics are to be performed to resolve the unknown magnetoviscous effects and slug internal flow. The simulation was also dependent on the initial position of the slug. Figure 4 shows the relationship between the maximum pressure differential generated and initial position of the center of the slug relative to the solenoid. The solid line represents the simulation with an effective length of $10.5 \mathrm{~cm}$ and a viscosity 2.1 times the non-magnetized value. The experimental data was for a visible slug length of $1.9 \mathrm{~cm}$ and the points marked with an " $\mathrm{x}$ " indicate runs that experienced a hydrostatic breakdown. Theoretical predictions validated by Perry [14] indicate that a breakdown should have occurred around $1.9 \mathrm{kPa}(0.27 \mathrm{psi})$ for the current setup, slightly higher than the data obtained. The difference was again due to the resistance heating.

The simple model was used to predict peak pressure trends with respect to slug length and position. Figure 5 shows the simulation's predictions on the maximum generated pressure differential as the slug length and center position vary. The simulation showed that the maximum pressure differential occurred when the slug was about twice the length of the solenoid and began with its closest edge just outside the solenoid. In a system free of shear, the pressure differential would continue to increase with the slug length and position.

As a higher order test, a $2.3 \mathrm{~cm}$ slug was pulsed between two solenoids at 0.25 seconds each from upstream to downstream. Figure 6 shows the correlation for the experimental data when using an effective slug length of $11.1 \mathrm{~cm}$ and a viscosity 2.1 times the non-magnetized value. This result proved that the LOX slug could be moved from solenoid to solenoid indicating that multiple solenoids could be used to move and control the motion of a LOX slug for certain applications. The results of this study further indicate that, in order to further understand the phenomena and optimize the simulation, the effect of magnetic field on the viscosity of LOX must be determined. Furthermore a 3D model of the 2-phase problem coupled with fluid flow and magnetics is needed to provide more information on the internal flow dynamics of the LOX slug.

\section{CONCLUSIONS}

Experimental testing of the magnetohydrodynamics of a LOX slug in a closed system has been achieved. A numerical model was developed to qualitatively simulate the 
dynamics when the slug is displaced by pulsed magnetic fields; however, further research is needed to more accurately match the model to experimental data. The magnetic fields are induced by sequencing current through a series of solenoids that control the slug by generating pressure pulses for compression and decompression. For the test apparatus presented, the pressure pulses were on the order of $2 \mathrm{kPa}$, comparable to other researchers using ferrofluids. The simulation has proven effective in gaining understanding of the pressure differential generated for a range of slug lengths and starting positions. The study provided insight into slug motion due to a single solenoid and a series of solenoids, thus paving the way for more complex research. Overall, the understanding achieved on the magnetohydrodynamic response of a LOX slug through experimentation and simulation will be useful for future researchers seeking to design subsystems with no moving parts.

\section{ACKNOWLEDGEMENTS}

This material is based on the research sponsored by the Air Force Research Laboratory, under agreement number FA9550-08-1-0018. The U.S. Government is authorized to reproduce and distribute reprints for Governmental purposes notwithstanding any copyright notation thereon. The views and conclusions contained herein are those of the authors and should not be interpreted as necessarily representing the official policies or endorsements, either expressed or implied, of the Air Force Research Laboratory or the U.S. Government. Dr. Clair Batty is credited for project synthesis of the study and Dr. Steven Hansen, Dr. Byard Wood, Dr. Doran Baker, and Ms. Gayle Bowen are thanked for arranging financial provisions through the Rocky Mountain NASA Space Grant Fellowship and Tomorrow Fellowship.

\section{REFERENCES}

1. Rosensweig, R. E., Ferrohydrodynamics, Dover, New York, 1985, pp. 13-123.

2. Park, G. S., and Park, S. H., "Design of Magnetic Fluid Linear Pump," IEEE Transactions on Magnetics, 35, pp. 4058-4060 (1999).

3. Park, G. S., and Park, S. H., "New Structure of the Magnetic Fluid Linear Pump," IEEE Transactions on Magnetics, 36, pp. 3709-3711 (2000).

4. Park, G. S., and Seo, K., "A Study on the Pumping Forces of the Magnetic Fluid Linear Pump," IEEE Transactions on Magnetics, 39, pp. 1468-1471 (2003).

5. Park, G. S., and Seo, K., "New Design on the Magnetic Fluid Linear Pump to Reduce the Discontinuities of the Pumping Forces," IEEE Transactions on Magnetics, 40, pp. 916-919 (2004).

6. Seo, K. and Park, G. S., "A Research on the Pumping Forces in the Magnetic Fluid Linear Pump," IEEE Transactions on Magnetics, 41, pp. 1580-1583 (2005).

7. Krauss, R. and Liu, M., "Pumping Fluid by Magnetic Surface Stress,” New J. of Physics, 8, pp. 1-11 (2006).

8. Hatch, A. and Kamholz, A. E, “A Ferrofluid Magnetic Micropump,” J. of Microelectromech. Syst., 10, pp. 215-221 (2001).

9. Liao, W. and Chen, X., "Tunable Optical Fiber Filters with Magnetic Fluids," Applied Physics Letters, 87, pp. 1-3 (2005).

10. Youngquist, R. C. and Immer, C. D., "Dynamics of a Finite Liquid Oxygen Column in a Pulsed Magnetic Field," IEEE Transactions on Magnetics, 39, pp. 2068-2073 (2003).

11. White, F. M., Viscous Fluid Flow, McGraw-Hill International Editions, Singapore, 1991, pp. 422-424.

12. Anderson, B. W., The Analysis and Design of Pneumatic Systems, Robert E. Krieger Publishing Company, Huntington, New York, 1976, pp. 103-105.

13. Odenbach, S., Magnetoviscous Effects in Ferrofluids, Springer-Verlag, Berlin, 2002, pp. 33-121.

14. Perry, M. P. and Jones, T. B., "Hydrostatic Loading of Magnetic Liquid Seals," IEEE Transactions on Magnetics, 12, pp. 798-800 (1976). 